\title{
Suppression of Streptococcus mutans and Candida albicans by Probiotics: an In vitro Study
}

\section{Shyamali Saha ${ }^{1,2}$, Catherine Tomaro-Duchesneau ${ }^{1}$, Meenakshi Malhotra ${ }^{1}$, Maryam Tabrizian ${ }^{1,2}$ and Satya Prakash ${ }^{1 *}$}

${ }^{1}$ Departments of Biomedical Engineering, Biomedical Technology and Cell Therapy Research Laboratory, Physiology, and Artificial Cells and Organs Research Center, Faculty of Medicine, McGill University, 3775 University Street, Montreal, Quebec, H3A 2B4, Canada

${ }^{2}$ Faculty of Dentistry, McGill University, Montreal, Quebec, H3A 2B2, Canada

\begin{abstract}
Oral infections caused by microorganisms have led to increased risk of oral health problems such as Dental Caries (DC), periodontitis and Oral Candidiasis (OC). Streptococcus mutans and Candida albicans are the primary organisms responsible for DC and OC, respectively. The goal of the presented study was to investigate the potential of probiotics to prevent and treat DC and OC. An in vitro assay was developed to investigate several probiotic strains for their ability to inhibit the aforementioned oral pathogens. Probiotic by-products present in probiotic supernatant and live probiotic cells were both investigated for their ability to inhibit the growth of S. mutans and C. albicans. The probiotic strains investigated were $L$. reuteri NCIMB 701359, L. reuteri NCIMB 701089, L. reuteri NCIMB 11951, L. reuteri NCIMB 702656, L. reuteri NCIMB 702655, L. fermentum NCIMB 5221, L. fermentum NCIMB 2797, L. fermentum NCIMB 8829, L. acidophilus ATCC 314, L. plantarum ATCC 14917 and L. rhamnosus ATCC 5310.The presented research demonstrates that live probiotic cells are needed to inhibit oral pathogens, as cell-free supernatant could not inhibit the pathogens. Further experiments were performed to investigate and optimize the dose-dependent inhibition of the pathogens by live probiotic cells. As desired, an increased inhibition was observed with an increase in dose, as demonstrated by the increasing size of the zones of clearance. In addition, the observed inhibition was dependent on the strain of the probiotic used. This research implies that probiotic bacteria are capable of inhibiting the selected oral pathogens, S. mutans and C. albicans, holding promise for the future development of a probiotic therapeutic to treat and prevent oral/dental diseases. Furthermore, the research proposes further investigations into the probiotic mechanism(s) of action and efficacy for the development of an optimal therapy.
\end{abstract}

Keywords: Oral disease; Dental caries; Oral candidiasis; Probiotic; $S$. mutans; C. albicans; Lactobacillus

\section{Introduction}

Dental Caries (DC), periodontitis and Oral Candidiasis (OC) are widespread disorders. According to the World Health Organization, $60-90 \%$ of worldwide school-children [1] and $95 \%$ of adults in the United States have DC [2]. Furthermore, $5-10 \%$ of public health expenditures relate to oral health - a significant economic burden [2]. It has been demonstrated that the initiation and progression of oral disease is primarily due to the increased proliferation of opportunistic microorganisms [3]. A large variety of microorganisms are associated with oral disease, however, the increased proliferation of $S$. mutans, present in carious lesions, is the primary cause for the initiation and the progression of DC, and hence is of primary interest in the presented research $[3,4]$. The relationship between this causative organism and pathogenesis is not clear, although it has been suggested that $S$. mutans proliferation leads to acid production, promoting tooth decay and DC [5]. OC, also termed thrush, is a yeast infection of the genus Candida, most often caused by C. albicans, the second pathogenic microorganism of focus in this research $[3,6,7]$.

The current methods for minimizing the incidence of DC focus on prevention techniques such as proper oral hygiene, drinking water fluoridation and application of dental sealants [8]. Unfortunately, as the statistics suggest, these prevention methods have shown only limited success. OC is typically controlled by anti-fungal drugs such as amphotericin B [9]. If not established as a biofilm, C. albicans is usually susceptible to most anti-fungals, but mechanical disruption of a biofilm prior to anti-fungal application may be required. A well-known problem is that long-term exposure to anti-fungal agents promotes acquired resistance [10]. The documented levels of resistance in oral candida are indeed on the rise [10]. To address these shortcomings, an alternative therapy is necessary for controlling the incidence of DC and OC, without the need for antimicrobials. Recent research has elucidated the role and importance of the oral microflora in pathogenesis $[7,11]$. With this in mind, investigators have proposed probiotics, specifically Lactobacilli, for maintaining and regaining oral health [12]. Lactobacilli have been used as probiotics for a number of disease applications, and are generally regarded as safe for human use, making them an ideal candidate for the development of a therapeutic [13,14].

This work investigates probiotic Lactobacillus strains that have potential of inhibiting oral pathogens, specifically $S$. mutans NCIMB 702062 and C. albicans ATCC 11006. The probiotic strains investigated were L. rhamnosus ATCC 53103, L. fermentum NCIMB 5221, L. fermentum NCIMB 2797, L. fermentum NCIMB 8829, L. reuteri NCIMB 11951, L. reuteri NCIMB 702656, L. reuteri NCIMB 702655 , L. reuteri NCIMB 701359, L. reuteri NCIMB 7010 89, L. acidophilus ATCC 314 and L. plantarum ATCC 14917.

\section{Materials and Methods}

\section{Bacterial growth media and chemicals}

De Man, Rogosa, Sharpe (MRS) broth was purchased from Fisher Scientific (Ottawa, ON, Canada). Water was purified with an Easy

*Corresponding author: Satya Prakash, Departments of Biomedical Engineering Biomedical Technology and Cell Therapy Research Laboratory, Physiology, and Artificial Cells and Organs Research Center, Faculty of Medicine, McGill University 3775 University Street, Montreal, Quebec, H3A 2B4, Canada, Tel: 1514-398-3676 Fax: 1514-398-7461; E-mail: satya.prakash@mcgill.ca

Received July 25, 2012; Accepted August 21, 2012; Published August 23, 2012

Citation: Saha S, Tomaro-Duchesneau C, Malhotra M, Tabrizian M, Prakash S (2012) Suppression of Streptococcus mutans and Candida albicans by Probiotics: an In vitro Study. Dentistry 2:141. doi:10.4172/2161-1122.1000141

Copyright: @ 2012 Saha S, et al. This is an open-access article distributed under the terms of the Creative Commons Attribution License, which permits unrestricted use, distribution, and reproduction in any medium, provided the original author and source are credited. 
Pure reverse osmosis system and a Nano Pure Diamond Life Science (UV/UF) ultrapure water system from Barnstead (Dubuque, IA, USA). All other chemicals used were of HPLC or standard analytical grade purchased from Sigma-Aldrich (Oakville, ON, Canada).

\section{Bacterial strains and culture conditions}

L. rhamnosus ATCC 53103, L. acidophilus ATCC 314, L. plantarum ATCC 14917 and Escherichia coli ATCC 8739 were purchased from Cedarlane Laboratories (Burlington, ON, Canada). L. fermentum NCIMB 5221, L. fermentum NCIMB 2797, L. fermentum NCIMB 8829, L. reuteri $\mathrm{NCIMB} 701089$, L. reuteri $\mathrm{NCIMB} 701359$, L. reuteri $\mathrm{NCIMB}$ 702655, L. reuteri NCIMB 702656 and L. reuteri NCIMB 11951 were purchased from NCIMB (Aberdeen, Scotland, UK). S. mutans NCIMB 702062, isolated from carious dentine, was purchased from NCIMB (Aberdeen, Scotland, UK) and C. albicans ATCC 11006 was generously gifted by Micropharma Limited (Montreal, Canada). All the bacterial strains were maintained at $-80^{\circ} \mathrm{C}$ as $20 \%(\mathrm{v} / \mathrm{v})$ glycerol stocks. A MRS agar plate was streaked and incubated at $37^{\circ} \mathrm{C}$ for 24 hours with $5 \%$ $\mathrm{CO}_{2}$ to ensure the purity of the culture. One colony from the MRS agar plate was inoculated in MRS broth for 24 hours at $37^{\circ} \mathrm{C}$. A $1 \%(\mathrm{v} / \mathrm{v})$ inoculum was subcultured into MRS broth and incubated at $37^{\circ} \mathrm{C}$ for 24 hours for the assays.

\section{Inhibition of $S$. mutans by probiotic supernatant and live cells}

S. mutans was tested for inhibition by the probiotic supernatants and live cultures of the following probiotic strains: 1) L. rhamnosus ATCC 53103, 2) L. acidophilus ATCC 314,3$)$ L. plantarum ATCC $14917,4)$ L. fermentum NCIMB 5221, 5) L. fermentum NCIMB 2797, 6) L. fermentum NCIMB 8829, 7) L. reuteri NCIMB 701089, 8) L. reuteri NCIMB 701359,9$)$ L. reuteri NCIMB 702655,10$)$ L. reuteri NCIMB 702656 and 11) L. reuteri NCIMB 11951. MRS agar (15\% (w/v)) was prepared and autoclaved, after which it was cooled to $55^{\circ} \mathrm{C}$ in a water bath. S. mutans was incorporated in the molten agar at a concentration of $0.5 \%(\mathrm{v} / \mathrm{v})$ overnight culture. Agar plates were poured, each consisting of $30 \mathrm{~mL}$ and cooled to room temperature. Four wells were formed in each plate. To test the inhibition via probiotic by-product, the supernatant was used in two different ways. First, the supernatant was collected from each Lactobacillus strain by pelleting overnight and 48 hours probiotic cultures at $4000 \mathrm{rpm}$ for 30 minutes at $4^{\circ} \mathrm{C}(\mathrm{NAPCO}$ 2028R centrifuge). The supernatant was filtered using a $0.2 \mu \mathrm{m}$ filter and the $\mathrm{pH}$ was adjusted to 6.5 using $2 \mathrm{M} \mathrm{NaOH}$. The supernatants were prepared in 2-fold serial dilutions using sterile MRS broth: 1) $100 \%(\mathrm{v} / \mathrm{v}), 2) 50 \%(\mathrm{v} / \mathrm{v}) 3) 25 \%(\mathrm{v} / \mathrm{v})$ and 4$) 12.5 \%$ of the original supernatant concentration. Secondly, the supernatant was filtered using a $0.2 \mu \mathrm{m}$ filter and 2 -fold serial dilutions were prepared using sterile MRS broth as aforementioned, without adjusting the $\mathrm{pH}$.

Similarly, for tests with probiotic live cultures, 10-fold serial dilutions of the probiotic overnight cultures were prepared in sterile MRS broth: 1) $100 \%(\mathrm{v} / \mathrm{v})$ 2) $10 \%(\mathrm{v} / \mathrm{v})$ 3) $1 \%(\mathrm{v} / \mathrm{v})$ and 4$) 0.1 \%$ $(\mathrm{v} / \mathrm{v})$. The wells in each plate were filled with $100 \mu \mathrm{l}$ of the respective diluted probiotic overnight strain culture/ supernatant at desired concentration. Sterile MRS broth and E. coli ATCC 8739 were used as negative controls. The plates were incubated at $37^{\circ} \mathrm{C}$ and $5 \% \mathrm{CO}_{2}$ for $48 \mathrm{hrs}$. Each probiotic strain was tested in triplicate to ensure accuracy and reproducibility and controls were treated exactly the same way as test samples. Following 48 hours, the diameters of the inhibition zones were measured for each well.

\section{Inhibition of C. albicans via live probiotics and probiotic by- products}

The probiotic by product and live culture mediated inhibition of C. albicans was investigated using the methods described above for $S$. mutans with slight modifications. C. albicans was incorporated in the molten agar at a concentration of $1 \%(\mathrm{v} / \mathrm{v})$ of both overnight and 48 hour culture. The same probiotic strains, described above for $S$. mutans, were tested for the probiotic-mediated inhibition of C. albicans.

\section{Bacterial viability}

The viability of the pathogenic and probiotic bacteria was measured using standard colony forming units (cfu). $0.85 \%(\mathrm{w} / \mathrm{v}) \mathrm{NaCl}$ was used to achieve 10-fold serial dilutions of each strain, as required. Each strain was streaked on MRS-agar plates and incubated at $37^{\circ} \mathrm{C}$ and $5 \%$ $\mathrm{CO}_{2}$ for 48 hours, prior to colony counting. Each strain was plated in triplicate to ensure accuracy and reproducibility.

\section{Statistical analysis}

Experimental results are expressed as mean \pm standard error of the mean (SEM). Statistical analysis was carried out using SPSS Version 17.0 (Statistical Product and Service Solutions, IBM Corporation, New York, NY, USA). Linear regression was performed to correlate probiotic cell count and zone of clearance.

\section{Results}

\section{S. mutans and C. albicans inhibition via probiotic by-products}

There were no visible zones of inhibition of S. mutans ATCC 702062 and C. albicans ATCC 11006 for any of the supernatant probiotics and controls tested (results not shown): L. reuteri NCIMB 701359, L. reuteri NCIMB 701089, L. reuteri NCIMB11951, L. reuteri NCIMB 702656, L. reuteri NCIMB 702655, L. fermentum NCIMB 5221, L. fermentum NCIMB 2797, L. fermentum NCIMB8829, L. acidophilus ATCC 314, L. plantarum ATCC 14917 and L. rhamnosus ATCC 53103.

\section{S. mutans inhibition using live probiotic cultures}

The inhibition of $S$. mutans ATCC 702062 was visible by clearing zones in the MRS agar around the wells containing the probiotic strains. The viability of the incorporated $S$. mutans was $1.04 \times 10^{7} \mathrm{cfu} /$ $\mathrm{ml}$ of agar. The inhibition zones, produced by probiotic activity, were clearly visible, as seen in Figure 1. L. reuteri NCIMB 701359, L. reuteri NCIMB 701089, L. reuteri NCIMB11951, L. reuteri NCIMB 702656, L. reuteri NCIMB 702655, L. fermentum NCIMB 5221, L. fermentum NCIMB 2797, L. fermentum NCIMB8829, L. acidophilus ATCC 314, L. plantarum ATCC 14917and L. rhamnosus ATCC 53103 were all able to inhibit the growth of $S$. mutans, with varying inhibition activity for each strain. Clearance zones were measured from the edge of the wells to the edge of the clearance zones. Pathogenic microorganism clearance zones were quantified using Image J (Rasband, W.S., Image J, U. S. National Institutes of Health, Bethesda, Maryland, USA, http:// imagej.nih.gov/ij/, 1997-2011). The negative controls (sterile MRS broth) and E. coli ATCC 8739, showed no inhibition, suggesting a probiotic-specific inhibition.

\section{C. albicans inhibition using live probiotic cultures}

The inhibition of C. albicans ATCC 11006 was visibly demonstrated by clearance zones in the MRS agar around the wells containing the probiotics. The viability of the incorporated C. albicans was $3.67 \mathrm{x}$ $10^{4} \mathrm{cfu} / \mathrm{ml}$ of agar. The inhibition zones, due to probiotic activity, were visible, as seen in Figure 2. L. reuteri NCIMB 701359, L. reuteri 
Citation: Saha S, Tomaro-Duchesneau C, Malhotra M, Tabrizian M, Prakash S (2012) Suppression of Streptococcus mutans and Candida albicans by Probiotics: an In vitro Study. Dentistry 2:141. doi:10.4172/2161-1122.1000141
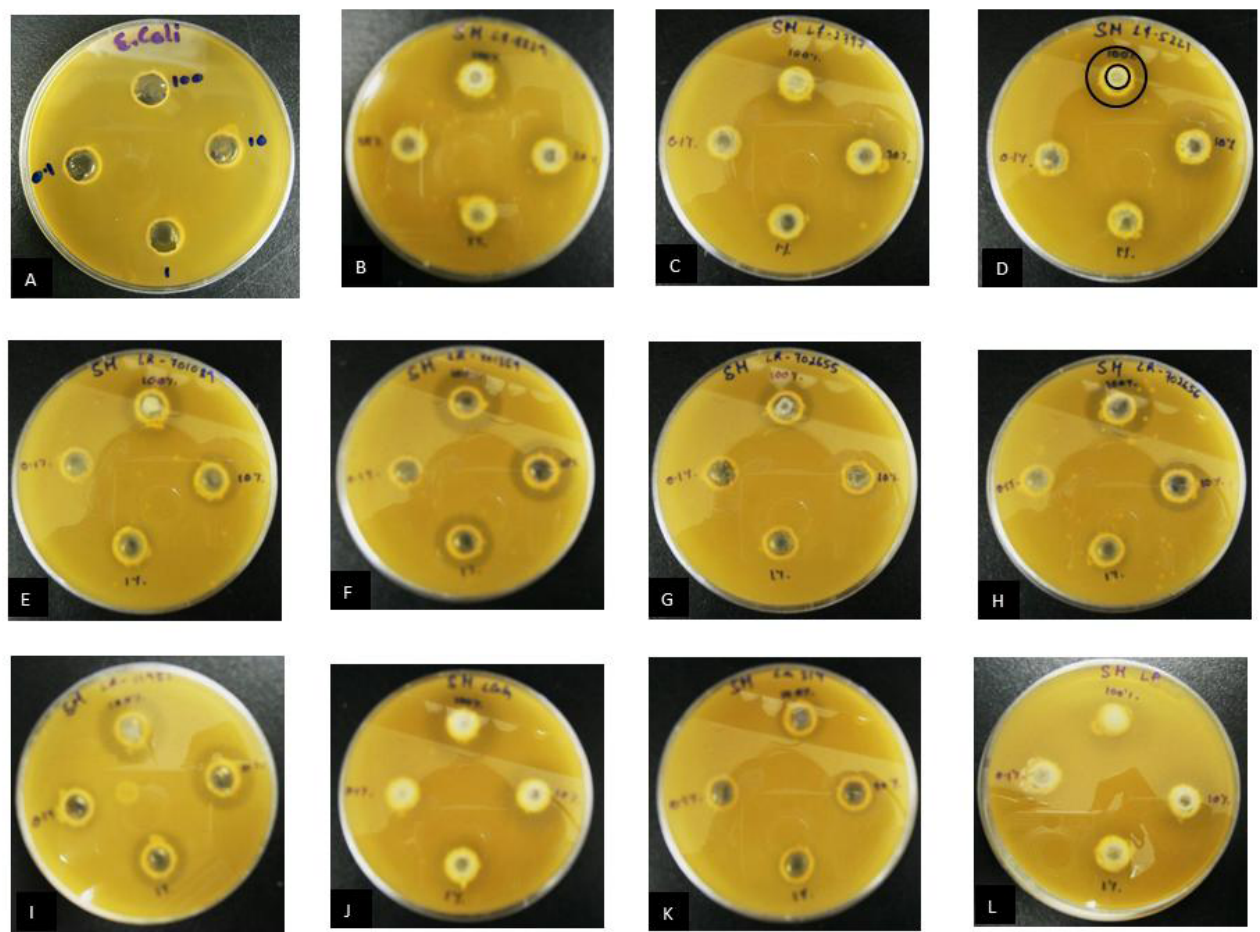

Figure 1: Inhibition assay for S. mutans ATCC 702062 demonstrating the zone of inhibition around the wells containing: (A) Negative Control $-E$. coli ATCC 8739; (B) Test strain L. fermentum NCIMB 8829; (C) Test strain L. fermentum NCIMB 2797; (D) Test strain L. fermentum NCIMB 5221; (E) Test strain L. reuteri NCIMB 701089; (F) Test strain L. reuteri NCIMB 701359; (G) Test strain L. reuteri NCIMB 702655; (H) Test strain L. reuteri NCIMB 702656; (I) Test strain L. reuteri NCIMB 11951; (J) Positive control- $L$. rhamnosus ATCC53103; (K) Test strain L acidophilus ATCC314; (L) Test strain L. plantarum ATCC14917 and *shows the clearance zone. The four wells contained the probiotic overnight culture of desired concentration diluted using sterile MRS:100\% (v/v), 10\% (v/v), 1\% (v/v) and 0.1\% (v/v). The agar plates were incubated for 48 hours at $37^{\circ} \mathrm{C}$ and $5 \% \mathrm{CO}_{2}$.
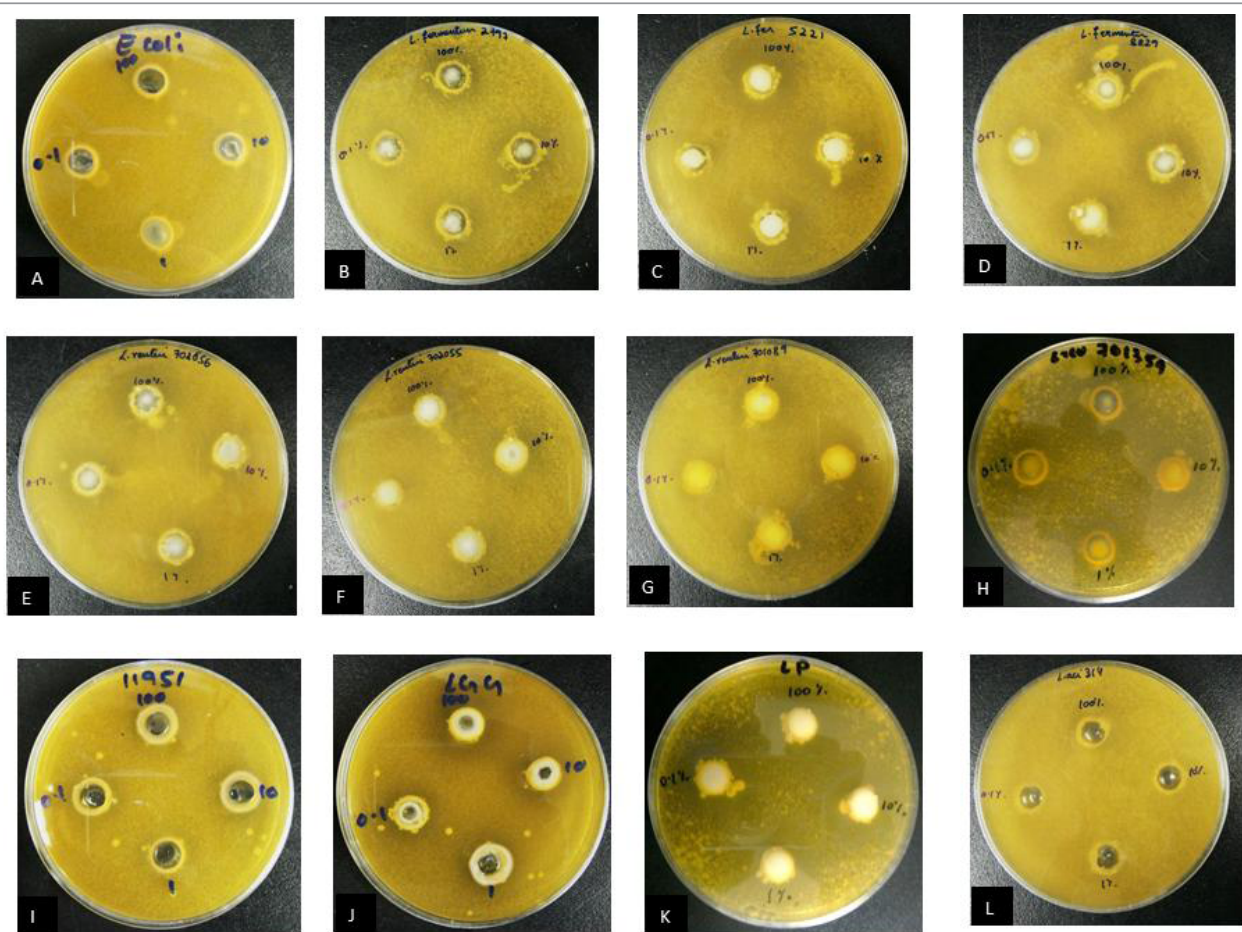

Figure 2: Inhibition assay for C. albicans ATCC demonstrating the zone of inhibition around the wells containing: (A) Negative Control -E. coli ATCC 8739; (B) Test strain L. fermentum NCIMB 8829; (C) Test strain L. fermentum NCIMB 2797; (D) Test strain L. fermentum NCIMB 5221; (E) Test strain L. reuteri NCIMB 701089; (F) Test strain L. reuteri NCIMB 701359; (G) Test strain L. reuteri NCIMB 702655; (H) Test strain L. reuteri NCIMB 702656; (I) Test strain L. reuteri NCIMB 11951; (J) Positive control- L. rhamnosus ATCC53103; (K) Test strain L. acidophilus ATCC314; (L) Test strain L. plantarum ATCC14917 and "shows the clearance zone. The four wells contained the probiotic overnight culture of desired concentration diluted using sterile MRS: $100 \%(\mathrm{v} / \mathrm{v}), 10 \%(\mathrm{v} / \mathrm{v}), 1 \%(\mathrm{v} / \mathrm{v})$ and $0.1 \%(\mathrm{v} / \mathrm{v})$. The agar plates were incubated for 48 hours at $37^{\circ} \mathrm{C}$ and $5 \% \mathrm{CO}_{2}$. 
NCIMB 701089, L. reuteri NCIMB 11951, L. reuteri NCIMB 702656, L. reuteri NCIMB 702655, L. fermentum NCIMB 5221, L. fermentum NCIMB 2797, L. fermentum NCIMB 8829, L. acidophilus ATCC 314, L. plantarum ATCC 14917 and L. rhamnosus ATCC 53103 all inhibited the growth of C. albicans, with varying activity for each strain, as seen in Figure 2. Clearance zones were measured for all the samples. The negative controls with sterile MRS and E. coli ATCC 8739 showed no zones of inhibition, demonstrating probiotic-specific inhibition of $C$. albicans.

\section{Discussion}

OC and DC are disorders for which the aetiology has been directly correlated with the presence and proliferation of pathogenic microorganisms. Various microorganisms are associated with the initiation and progression of dental caries, among which S. mutans and S. sobrinus are the most prominent caries causing agents [15]. However, number of research groups has demonstrated that $S$. mutans is more frequently associated with DC than $S$. sobrinus, hence the focus is on the former in this work [15-18]. As for OC, C. albicans is the principle causative organism $[18,19]$.

Probiotic bacteria have been used to modify microfloral ecosystems, and have already shown some success as a therapeutic for oral diseases, as described in a recent review [20]. For the development of a more efficacious therapeutic, optimization and selection of probiotic strains need to be undertaken. The goal of the presented research was to screen probiotic strains, chosen based on already demonstrated beneficial properties in other human disorders, to investigate their potential to inhibit the two prominent oral pathogens, S. mutans and C. albicans via probiotic by-products and live probiotic cultures.

Inhibition assays were performed using two probiotic formulations: 1) cell supernatant (by-products) and 2) live cells. An in vitro assay was designed to investigate the probiotic inhibition of $S$. mutans NCIMB 702062 and C. albicans ATCC 11006. The S. mutans used in this investigation was isolated from carious dentine and was used as a proof of concept for other pathogenic strains. C. albicans was used in its yeast form as opposed to hypha form as that is the phenotype involved in the clinical manifestation of oral candidiasis [21]. The inhibition assay consisted of MRS agar embedded with the oral pathogens, with preformed wells in the agar containing the supernatant or live cells. Clearance zones demonstrated the ability of the supernatant or live probiotic formulation to inhibit the growth of the pathogens. Previous research has demonstrated that probiotic supernatant filtered of the cell contents showed inhibition of oral biofilm due to bacteriocin activity, such as reuterin, originating from $L$. reuteri [22]. Hence, the first experimental approach undertaken was to use probiotic supernatant to determine whether a secreted factor produced during culturing could inhibit the oral pathogens that contribute to biofilm formation. The results demonstrated no detectable inhibition for either C. albicans or S. mutans. The overnight probiotic cultures were further grown for an extended period of 48 hours to confirm that probiotic supernatants contained enough secreted products for optimum function. However, none of the probiotic supernatants were able to demonstrate any inhibition of S. mutans or C. albicans. As aforementioned, the $\mathrm{pH}$ of the supernatant from all the tested probiotic bacterial strains was adjusted to 6.7 by the addition of $\mathrm{NaOH}$. Hence, to rule out the effect of $\mathrm{NaOH}$, both 24 and 48 hours of bacterial supernatants were also tested without the addition of $\mathrm{NaOH}$. The rationale behind these two approaches was first to evaluate the effect of secreted products from the probiotics, by eliminating the possibility of an observed inhibition due to the effect of
$\mathrm{pH}$ of the tested supernatants and second, to eliminate the possibility of inactivating/modifying the functionality of the probiotic supernatant by the addition of $\mathrm{NaOH}$. An explanation for the lack of inhibition by probiotic supernatant may be due to a number of factors, for example, organic acids or bacteriocins produced by the probiotic may be unstable or degraded or are simply present in too low concentrations. However, when the live probiotic cell cultures were used, all of the tested strains proved capable of inhibiting the proliferation of S. mutans and. C. albicans, with the zones of inhibition variable for each probiotic strain. Further experiments were then performed to evaluate the capability and dose response of different live probiotic cell concentrations to inhibit S. mutans and C. albicans. As expected, a positive correlation between the area of the zones of clearance and the concentration of probiotic cells was observed.

S. mutans and C. albicans were incorporated at $0.5 \%(\mathrm{w} / \mathrm{v})$ and $1 \%$ $(\mathrm{w} / \mathrm{v})$ respectively. This corresponded to bacterial concentrations of $1.04 \times 10^{7} \pm 7.04 \times 10^{5}$ for $S$. mutans and $3.67 \times 10^{4} \pm 1.67 \times 10^{4}$ for $C$. albicans. Due to the low bacterial count for C. albicans as compared to S. mutans, the former was incorporated in a higher concentration to yield enough growth for a visible inhibition by the probiotic strains. Zones of clearance were then evaluated and attempts were made to evaluate the cfu required for a $1 \mathrm{~mm}$ inhibition by normalizing the results. However, when the zones of clearance were plotted against the cfu, a weak linear correlation was demonstrated by some of the probiotic strains (indicated by their respective $\mathrm{R}^{2}$ values), as observed in Figure 3. The $p$-values were also generated for the linear regression analysis of zone of clearance versus probiotic viability for each probiotic strain tested [shown in Figure 3(a), 3(b), 3(c), 4(a), 4(b), and 4(c)]. As observed in Figure 3(a) and Figure 3(b), L. fermentum NCIMB 5221 and L. reuteri NCIMB 701359 demonstrated the largest zones of clearance as compared to the other tested strains, suggesting them as the most efficient strains for the inhibition of $S$. mutans ATCC 702062. Similar results were observed for the inhibition assay designed for C. albicans. As observed in Figure 4(a), L. fermentum NCIMB 5221 demonstrated the largest zone of clearance as compared to the other tested probiotic strains. Again, L. fermentum NCIMB 5221 appears to be the most effective probiotic amongst all the tested strains for the inhibition of C. albicans ATCC 11006.

In a similar study, Hasslof et al. [23] have demonstrated the inhibitory action on oral pathogens via probiotics. They investigated the ability of probiotic suspension but did not compare it to their cell free supernatants which could potentially have an effect on the aforementioned pathogens. As described, similar results were demonstrated in this study, moreover it could also be concluded that probiotic by-products were either inefficient at inhibiting $S$. mutans and C. albicans or were not stable or degraded to be able to functional optimally. In addition, this observation clues that if the inhibition of pathogens was not due to the substance releases by the probiotics, it could be due a competition of nutrients between the pathogens and the probiotics, for example for sucrose. In addition, ten different Lactobacillus strains were investigated for an efficient therapeutic for DC and OC and L. rhamnosus ATCC 53103 were used as a positive control from Hasslof et al. [23] study.

The mechanisms of action behind the observed inhibition need to be investigated further. In vivo, several mechanisms may be involved in a successful probiotic inhibition of pathogens, namely: 1) inhibition of pathogenic molecules 2) inhibition of exo-polysaccharide (EPS) secretion, responsible for dental plaque formation which allows for 
Citation: Saha S, Tomaro-Duchesneau C, Malhotra M, Tabrizian M, Prakash S (2012) Suppression of Streptococcus mutans and Candida albicans by Probiotics: an In vitro Study. Dentistry 2:141. doi:10.4172/2161-1122.1000141

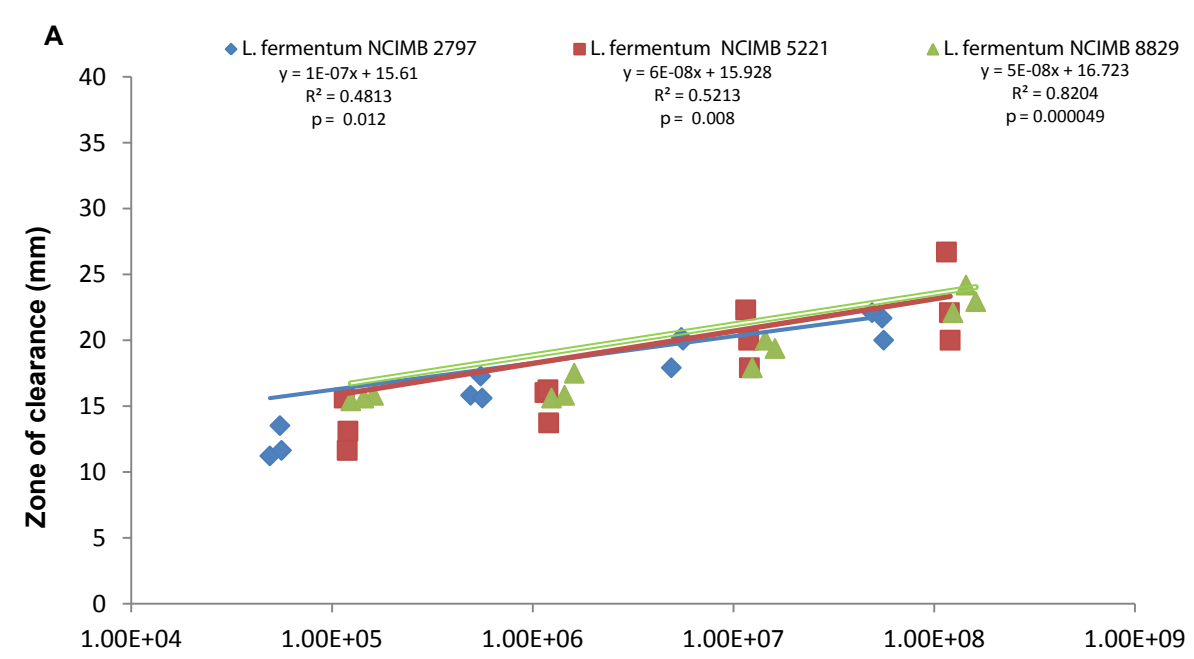

Bacterial viability (cfu)
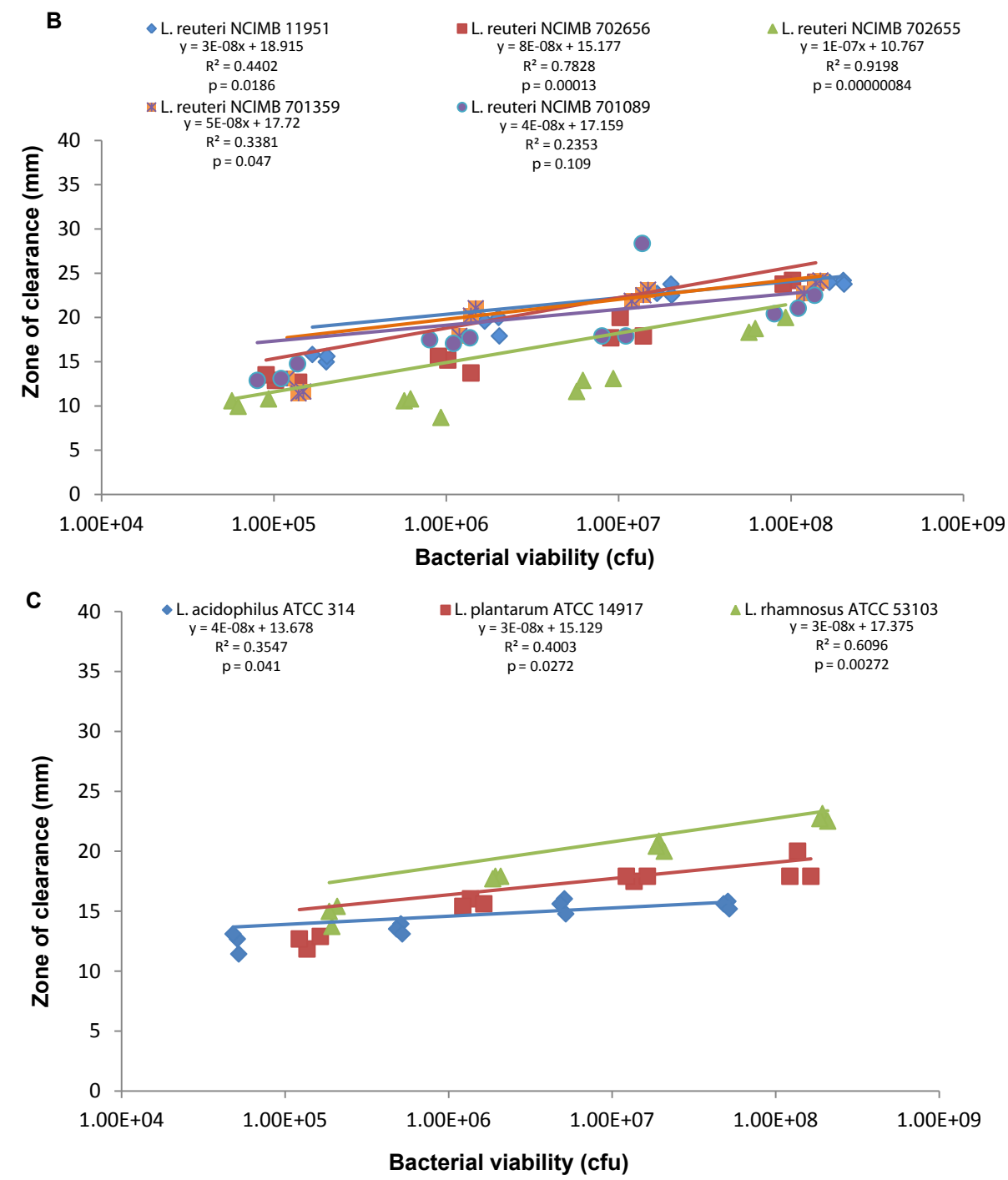

Figure 3: Inhibition of S. mutans ATCC 702062 by probiotic strains : A) L. fermentum NCIMB 2797, L. fermentum NCIMB 5221 and L. fermentum NCIMB 8829 ; B) L. reuteri NCIMB 11951, L. reuteri NCIMB 702655, L. reuteri NCIMB 702656, L. reuteri NCIMB 701359 and L. reuteri NCIMB 701089 . Data reflects individual triplicate points and linear regression was performed to correlate bacterial viability with zone of clearance, generating the $\mathrm{R}^{2}$ values and the associated $p$-values. 
Citation: Saha S, Tomaro-Duchesneau C, Malhotra M, Tabrizian M, Prakash S (2012) Suppression of Streptococcus mutans and Candida albicans by Probiotics: an In vitro Study. Dentistry 2:141. doi:10.4172/2161-1122.1000141

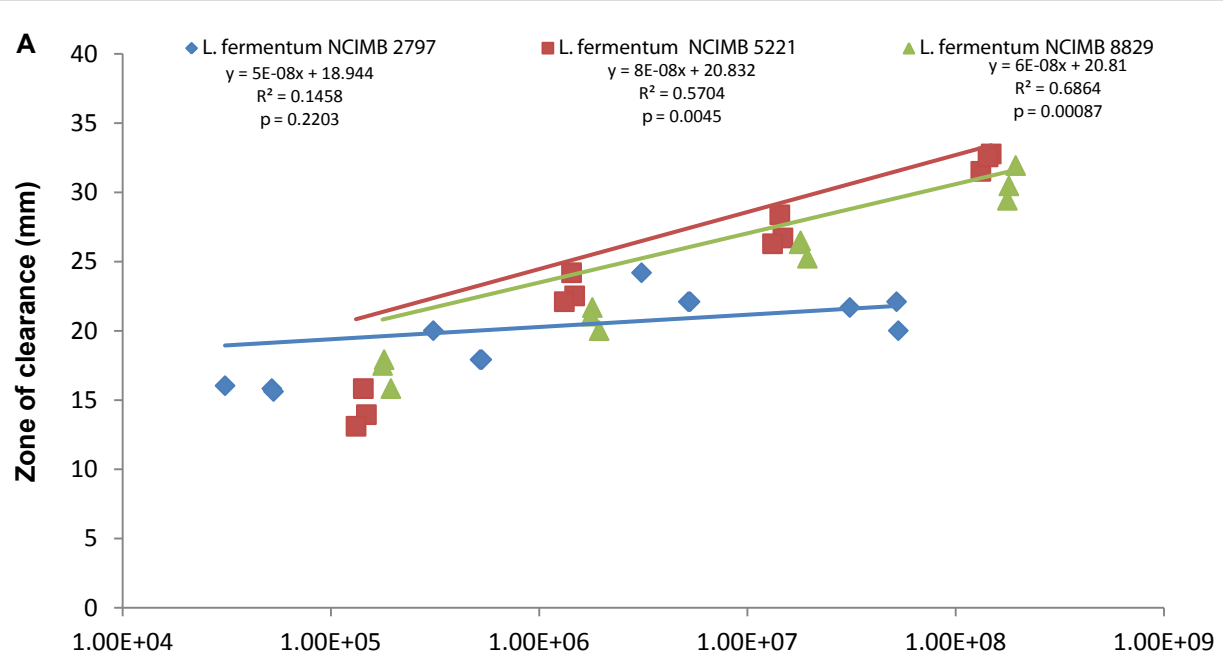

Bacterial viability (cfu)

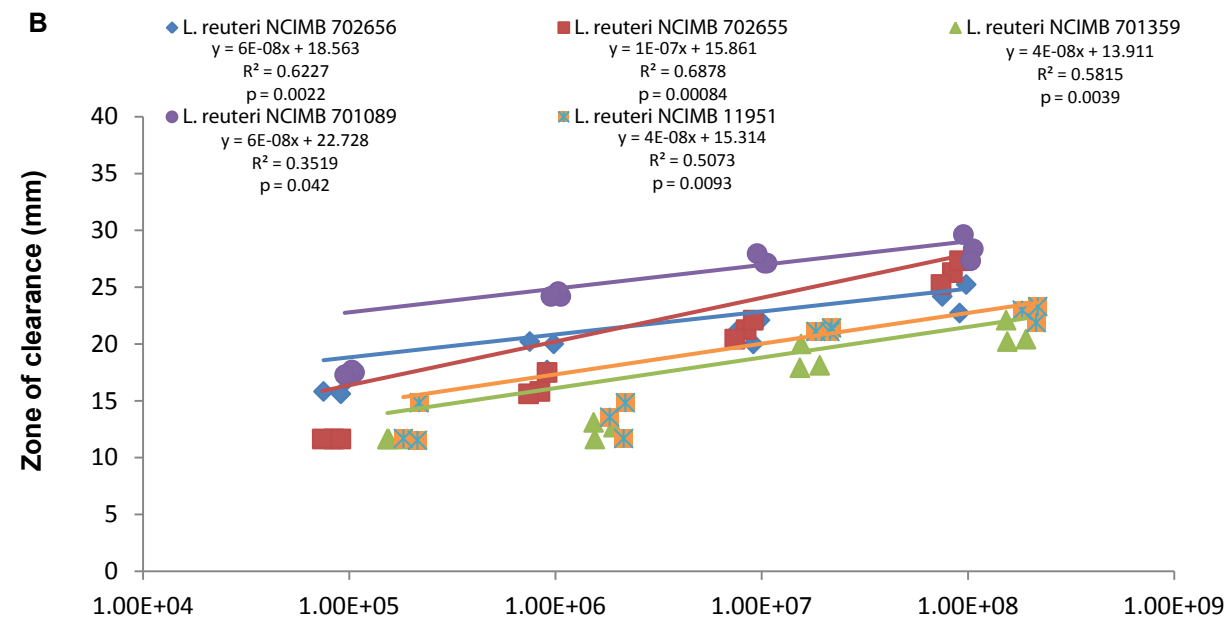

Bacterial viability (cfu)

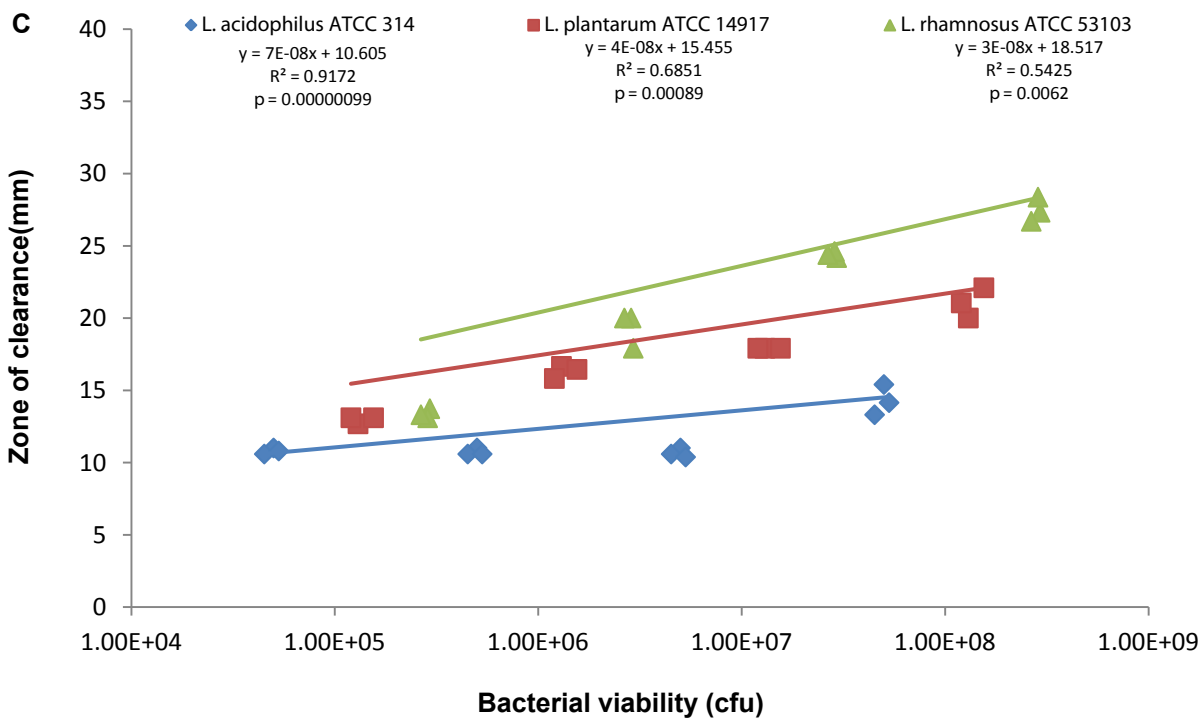

Figure 4: Inhibition of C. albicans ATCC 11006 by probiotic strains : A) L. fermentum NCIMB 2797, L. fermentum NCIMB 5221 and L. fermentum NCIMB 8829 ; B) L. reuteri NCIMB 11951, L. reuteri NCIMB 702655, L. reuteri NCIMB 702656, L. reuteri NCIMB 701359 and L. reuteri NCIMB 701089 . Data reflects individual triplicate points and linear regression was performed to correlate bacterial viability with zone of clearance, generating the $\mathrm{R}^{2}$ values and the associated $p$-values. 
accelerated growth of opportunistic organisms 3) blocking of pathogen adhesion sites 4) oral $\mathrm{pH}$ modulation and 5) nutrient competition as discussed in another review [20]. For example, EPS is a component of oral biofilm that is essential for the growth of oral pathogens [4]. A secreted probiotic product that could decrease the production of EPS could be beneficial for the prevention of biofilm formation and dental caries pathogenesis. Methods such as Fourier transform infrared spectrophotometry and high performance liquid chromatography can be used to quantify EPS levels, and hence may prove beneficial for future such investigations [24].

Moreover, research groups such as Noordin and Kamin [25] were successful in demonstrating that probiotics reduced the accumulation of dental plaque/oral biofilm. However, modulation of composition of the oral biofilm with an increased amount of non-cariogenic organisms and a decrease of pathogenic organisms could prove beneficial. Therefore, another approach to prevent/treat oral diseases via probiotic therapeutic could also be a modulation of oral biofilm. Hence, further research that demonstrates a decrease in a bacterial count of pure pathogenic strains and pathogenic strain incorporated in biofilm could prove beneficial. One concern with the inhibition of C. albicans and S. mutans is the possibility that the observed inhibition was simply due to environmental changes by the probiotic bacteria, more specifically acid production. It is to be noted that most of the tested probiotic cultures, following incubation had a significantly higher $\mathrm{pH}$ than the tested pathogens (data not shown). Furthermore, S. mutans is an acidogenic/aciduric bacterium that thrives under acidic conditions. We, therefore, hypothesise that the observed inhibition is not due to acid production by the probiotic bacteria, although further investigations are required into the exact mechanism of action for the inhibition [26]. This study is a semi-quantitative assay, which attempts to select probiotic bacteria capable of inhibiting two significant oral pathogens. It is clear, that further quantitative investigations are required for a better understanding of the kinetics of the observed inhibition. Future experiments must also focus on the inhibition of probiotics in simulated conditions, biofilms and broth cultures, a more quantitative approach. As required for the selection of the best probiotic strain among these and other strains, a more quantitative assay needs to be performed involving conditions resembling the oral ecosystem.

Although a starting point, this research establishes the fact that the formulation of a probiotic therapy has enormous potential to prevent or treat DC, OC and potentially other oral disorders. However, it is important to note that bacterial antagonism is not the only criteria for the development of a probiotic therapeutic, and so further investigations into the proposed mechanism(s) of actions are a must. Future work should involve the additional screening and characterisation of a final formulation for potential preclinical use, in terms of the mechanisms of action and safety of the probiotic strains with proper in vitro and in vivo studies. The successful delivery and residence time of probiotics in the oral cavity also remains an issue. Techniques such as the incorporation and immobilization of probiotics in chewing gums, food matrices and dissolvable films may prove useful $[27,28]$. There is also the need for further investigations into the probiotic mechanism(s) of action before any formulation optimization can be undertaken. Using this assay, future work may also investigate additional strains for the inhibition of all pathogens related to oral diseases, including those of the red complex involved in periodontal diseases [29].
In summary, the presented work successfully investigated probiotics capable of inhibiting S. mutans and C. albicans. The results demonstrate that live probiotics are required for this inhibition since cell-free probiotic supernatant was not able to inhibit the pathogens. This work opens up future potentials for the development of a probiotic replacement therapy for oral diseases.

\section{Acknowledgement}

The authors would like to acknowledge the Canadian Institute of Health Research (ClHR) grant (MPO 64308) to Dr. S. Prakash, FRSQ Doctoral Scholarship to Meenakshi Malhotra and a Doctoral Alexander Graham Bell Canada Graduate Scholarship from NSERC to Catherine Tomaro-Duchesneau.

\section{References}

1. World Health Organization (2012) Oral Health.

2. National Institute of Dental and Craniofacial Research and National Institutes of Health (2011) Dental Caries in Permanent (Adult) Teeth.

3. van Houte J (1994) Role of micro-organisms in caries etiology. J Dent Res 73: 672-681.

4. Banas JA (2004) Virulence properties of Streptococcus mutans. Front Biosc 9: $1267-1277$.

5. Lisa S (2007) The role of Streptococcus mutans and oral ecology in the formation of dental caries. Lethbrige Undergraduate Research Journal 2.

6. Klein RS, Harris CA, Small CB, Moll B, Lesser M, et al. (1984) Oral candidiasis in high-risk patients as the initial manifestation of the acquired immunodeficiency syndrome. N Engl J Med 311: 354-358.

7. Marsh PD (1994) Microbial ecology of dental plaque and its significance in health and disease. Adv Dent Res 8: 263-271.

8. U.S. National Library of Medicine and U.S. Department of Health and Human Services National Institutes of Health (2010) Dental cavities.

9. Rautemaa R, Ramage G (2011) Oral candidosis -- clinical challenges of a biofilm disease. Crit Rev Microbiol 37: 328-336.

10. Niimi M, Firth NA, Cannon RD (2010) Antifungal drug resistance of oral fungi. Odontology 98: 15-25.

11. Gross EL, Leys EJ, Gasparovich SR, Firestone ND, Schwartzbaum JA, et al (2010) Bacterial 16S sequence analysis of severe caries in young permanent teeth. J Clin Microbiol 48: 4121-4128.

12. Haukioja A (2010) Probiotics and oral health. Eur J Dent 4: 348-355.

13. Prakash S, Tomaro-Duchesneau C, Saha S, Cantor A (2011) The gut microbiota and human health with an emphasis on the use of microencapsulated bacterial cells. J Biomed Biotechnol 2011:981214.

14. Tomaro-Duchesneau C, Saha S, Malhotra M, Coussa-Charley M, Al-Salami H et al. (2012) Lactobacillus fermentum NCIMB 5221 has a greater ferulic acid production compared to other ferulic acid esterase producing Lactobacilli. Int $\mathrm{J}$ probio prebio 7 .

15. Ahn SJ, Lim BS, Lee SJ (2007) Prevalence of cariogenic streptococci on incisor brackets detected by polymerase chain reaction. Am J Orthod Dentofacial Orthop 131: 736-741.

16. Beighton D, Manji F, Baelum V, Fejerskov O, Johnson NW, et al. (1989) Associations between salivary levels of Streptococcus mutans, Streptococcus sobrinus, lactobacilli, and caries experience in Kenyan adolescents. J Dent Res 68: $1242-1246$.

17. Lindquist B, Emilson CG (1991) Dental location of Streptococcus mutans and Streptococcus sobrinus in humans harboring both species. Caries Res 25 : 146-152.

18. Loesche WJ (1986) Role of Streptococcus mutans in human dental decay. Microbiol Rev 50: 353-380.

19. Liljemark WF, Bloomquist C (1996) Human oral microbial ecology and denta caries and periodontal diseases. Crit Rev Oral Biol Med 7: 180-198.

20. Saha S, Tomaro-Duchesneau C, Tabrizian M, Prakash S (2012) Probiotics as oral health biotherapeutics. Expert Opin Biol Ther 12: 1207-1220. 
Citation: Saha S, Tomaro-Duchesneau C, Malhotra M, Tabrizian M, Prakash S (2012) Suppression of Streptococcus mutans and Candida albicans by Probiotics: an In vitro Study. Dentistry 2:141. doi:10.4172/2161-1122.1000141

21. Sangeorzan JA, Bradley SF, He X, Zarins LT, Ridenour GL, et al. (1994) Epidemiology of oral candidiasis in HIV-infected patients: Colonization, infection, treatment, and emergence of fluconazole resistance. Am J Med 97: 339-346.

22. Soderling EM, Marttinen AM, Haukioja AL (2011) Probiotic lactobacilli interfere with Streptococcus mutans biofilm formation in vitro. Curr Microbiol 62: 618622.

23. Hasslof P, Hedberg M, Twetman S, Stecksen-Blicks C (2010) Growth inhibition of oral mutans streptococci and candida by commercial probiotic lactobacilli-an in vitro study. BMC Oral Health 10: 18.

24. Vijayabaskar $P$, Babinastarlin $S$, Shankar T, Sivakumar T, Anandapandian KTK (2011) Quantification and characterisation of exopolysaccharides from Bacillus subtilis (MTCC 121). Advances in Biological Research 5: 71-76.

25. Noordin K, Kamin S (2007) The effect of probiotic mouthrinse on plaque and gingival inflammation. Annal Dent 14: 19-25.

26. Denepitiya L, Kleinberg I (1984) A comparison of the acid-base and aciduric properties of various serotypes of the bacterium Streptococcus mutans associated with dental plaque. Arch Oral Biol 29: 385-393.

27. Twetman S, Derawi B, Keller M, Ekstrand K, Yucel-Lindberg T, et al. (2009) Short-term effect of chewing gums containing probiotic Lactobacillus reuteri on the levels of inflammatory mediators in gingival crevicular fluid. Acta Odontol Scand 67: 19-24.

28. Vivekananda MR, Vandana KL, Bhat KG (2010) Effect of the probiotic Lactobacilli reuteri (Prodentis) in the management of periodontal disease: a preliminary randomized clinical trial. J Oral Microbiol 2.

29. Holt SC, Ebersole JL (2005) Porphyromonas gingivalis, Treponema denticola and Tannerella forsythia: the 'red complex', a prototype polybacterial pathogenic consortium in periodontitis. Periodontol 2000 38: 72-122. 